Recepción: 06 / 10 / 2016

Aceptación: 27 / 01 / 2017

Publicación: 18 / 05 / 2017

Ciencias económicas y empresariales

Artículo corto

\title{
El plan de marketing y su importancia para el posicionamiento de las empresas
}

The marketing plan and its importance for the positioning of the companies

O plano de marketing e sua importância para o posicionamento das empresas

\author{
Kelly J. Estrada-Realpe ${ }^{\mathrm{I}}$ \\ kelly.estrada@utelvt.edu.ec \\ Rugina Elidea-Quiñonez ${ }^{\mathrm{II}}$ \\ rugina.quinonez@utelvt.edu.ec \\ Luz M. Cifuentes-Quiñonez ${ }^{\text {II }}$ \\ luzmcifuentes@hotmail.com \\ Jorgeli Ayovi-Caicedo rv \\ ayovi_jorge@hotmail.es
}

Correspondencia: kelly.estrada@utelvt.edu.ec

\footnotetext{
' Magister en Docencia y Gerencia en Educación Superior, Diploma Superior en Gerencia Estratégica de Mercadeo, Licenciada en Publicidad y Mercadotecnia, Docente de la Universidad Luis Vargas Torres de Esmeraldas, Esmeraldas, Ecuador.

" Magister En Administración de Empresas, Ingeniera en Administración Pública, Docente de la Universidad Técnica "Luis Vargas Torres" de Esmeraldas, Esmeraldas, Ecuador

"II Magister en Docencia Mención Gestión en Desarrollo del Currículo, Magister en Administración de Empresas, Licenciado en Contabilidad y Auditoría, Doctora en Contabilidad y Auditoría, Técnica en Comunicación Radiofónica Para el Desarrollo, Docente en la Universidad Técnica Luis Vargas Torres De Esmeraldas, Esmeraldas, Ecuador

w Magister en Administración de Empresas, Diploma Superior en Docencia Universitaria, Ingeniero en Marketing, Docente de la Universidad Luis Vargas Torres de Esmeraldas, Esmeraldas, Ecuador.
} 




\section{Resumen}

La mercadotecnia se propone determinar quiénes son los clientes. Sus agentes toman la decisión de cuáles productos o servicios pueden satisfacer las necesidades de estos. El Plan de Mercadotecnia es un instrumento que sirve para dirigir y coordinar actividades en aras de lograr un objetivo específico. Aquí se particulariza en aquello que se desea lograr y se hace de forma clara y cuantificable. Todo lo planificado se contempla a corto, mediano y largo plazo, analizando el mercado meta actual y potencial. Esta propuesta se orientó hacia el servicio como factor clave para atraer y mantener clientes, dentro de la concepción general del plan de marketing.

Palabras clave: plan de mercadotecnia; servicios; administración de empresa.

\section{Abstract}

Marketing aims to determine who the customers are. Your agents make the decision on which products or services can meet their needs. The Marketing Plan is an instrument that serves to direct and coordinate activities in order to achieve a specific objective. Here it is particularized in what is wanted to be achieved and is done in a clear and quantifiable way. Everything planned is considered in the short, medium and long term, analyzing the current and potential target market. This proposal was oriented towards the service as a key factor to attract and maintain clients, within the general conception of the marketing plan.

Keywords: Marketing plan; services; business administration.

\section{Resumo}

O marketing visa determinar quem são os clientes. Seus agentes tomam a decisão sobre quais produtos ou serviços podem atender às suas necessidades. O Plano de Marketing é um instrumento que serve para direcionar e coordenar atividades para atingir um objetivo específico. Aqui é particularizado no que se deseja alcançar e é feito de forma clara e quantificável. Tudo o planejado é considerado a curto, médio e longo prazos, analisando o mercado-alvo atual e potencial. Esta proposta foi orientada para o serviço como um fator chave para atrair e manter clientes, dentro da concepção geral do plano de marketing.

Palavras chave: plano de marketing; serviços; administração de empresas. 


\section{Introducción}

La mercadotecnia es sin duda una de las áreas más importantes para generar una mejoría en cuanto al posicionamiento de todas las empresas. Constituye una herramienta para que los dirigentes de la misma puedan planear, establezcan metas, promuevan y distribuyan los bienes y servicios para satisfacer deseos y necesidades a los clientes actuales y potenciales (Fischer y Espejo, 2004).

La mercadotecnia centra su objetivo en determinar quiénes son sus clientes, quiénes con su punto de vista, toman la decisión de qué productos o servicios pueden satisfacer sus necesidades.

Esta orientación hacia el mercado meta y mediante una investigación de los elementos que hagan satisfacer sus deseos y necesidades, ayudan al establecimiento de estrategias dentro del plan de mercadotecnia para el impulso del posicionamiento.

La mercadotecnia presenta cuatro características en relación a los servicios que se mencionan a continuación.

\section{- $\quad$ Intangibilidad}

Existe una gran diferencia entre los productos físicos y los servicios ya que están más delimitados a no ser visibles y por lo tanto es de suma importancia la experimentación de ellos para conocer el grado de satisfacción.

\section{- Inseparabilidad}

Kotler, Bowen \& Makens (1997:83) enfatizan que, en la mayoría de los servicios de hospitalidad, el proveedor de los servicios así como el cliente deben estar presentes para que la transacción se lleve a cabo. Los empleados que establecen contacto con los clientes forman parte del producto. Esto significa que los clientes forman parte del producto.

\section{- Heterogeneidad.}

Este concepto se refiere a que los servicios suelen ser diferentes y varían de acuerdo a las condiciones en que se presentan, pero deben ser constantes de tal manera que, si un cliente llega al mismo establecimiento, el servicio no debe cambiar y mantener cierta congruencia con los demás. Todo esto con el esfuerzo de las personas involucradas en dar el servicio, desde la 
promoción de ventas hasta el momento de alojamiento o alimentación para asegurar una calidad monitoreada en todo momento.

\section{- Carácter perecedero}

Kotler (1997:84) señala que no se pueden almacenar los servicios y estos permanecen constantes. En algunos casos de acuerdo a la demanda y la oferta, puede o no cubrir el mercado, pero tampoco pierden la posibilidad de mantener en operación a la empresa.

Un plan de mercadotecnia es un documento que funciona como instrumento que ayuda a dirigir y coordinar actividades a realizarse para lograr un objetivo específico. En este objetivo se detalla que se desea lograr de forma clara y cuantificable. Las diferentes actividades se contemplan a corto, mediano y largo plazo en base al análisis de la empresa frente a su mercado meta actual y potencial.

"El plan de marketing proporciona la descripción de como la organización combinara el producto, la fijación de precios, la distribución y las decisiones de promoción para crear una oferta que resulte atractiva a los clientes. También trata de la implementación, el control y el ajuste continuo de estas decisiones" (Ferrel \& Hartline, 2012).

Fischer \& Espejo (2004:6) definen a la mercadotecnia como: "Aquella actividad humana dirigida a satisfacer necesidades, carencias y deseos a través de procesos de intercambio". Con base en esta definición nuestra investigación se orientará hacia el servicio como factor clave para atraer y mantener clientes, estimular las ventas y generar una aceptación de los servicios ante el mercado meta actual y potencial.

\section{Desarrollo}

\section{Principios fundamentales para una propuesta de un plan de marketing}

Coherencia: "La idea de que la aplicación de criterios que se apoyen mutuamente en esferas normativas conexas probablemente producirá una mayor armonía entre los objetivos y los resultados".

Adaptación: “Adaptación se basa en realizar alguna modificación en el producto o en la estrategia de marketing, que puede ir desde realizar un pequeño cambio hasta ofrecer una versión del 
producto diferente o seguir una política comercial sustancialmente distinta en el mercado exterior, con el objetivo de acomodar la oferta de la empresa a los gustos de los clientes y a las condiciones del mercado del país extranjero".

Superioridad Parcial: "Cualidad o situación de la persona o la cosa que tiene el grado o la posición suprema o más alta en una escala, y que solo afecta a una parte”.

El Plan de Marketing, es una herramienta que sirve para prever cual será el comportamiento comercial en la empresa durante un periodo de tiempo. Posee características que se deben tomar en cuenta:

- $\quad$ Es una herramienta de trabajo empresarial.

- $\quad$ Se diseña para que sea útil a la empresa.

- $\quad$ Es periódica, es decir, habitualmente su tiempo de desarrollo es para un año, aunque hay empresas que diseñan un Plan de Marketing para periodos más cortos (3 meses, 6 meses, según la actividad empresarial).

- El diseño ha de seguir un orden, sin esta premisa la planificación se convertirá en caos, con lo que perderá su efectividad.

- $\quad$ El Plan de Marketing ha de ser realista, fácil de seguir por todos en la empresa, con datos objetivos y toma de decisiones (estrategias, tácticas, medidas, presupuestos, etc.) ajustadas a mercado y a la empresa.

- $\quad$ Es un documento flexible, por lo que debe ser susceptible de ser modificado.

En este sentido, el Plan de Marketing, es una guía para la comercialización que hará recorrer el camino necesario para rentabilizar los productos y generar una imagen de estos y de la empresa. Es necesaria para todo tipo de empresa, sin distinción en tamaño, productos, servicios o filosofías. Se centra en los intereses de los clientes para ofrecerles el producto que más adecuado a sus gustos y necesidades. Debemos conocer qué quieren comprar nuestros clientes para comercializar eso mismo, y por supuesto hacerlo de manera rentable para la empresa.

Con la implantación del Plan Marketing la organización persigue los siguientes objetivos: 
- $\quad$ Aumento de la cifra de negocios y de los beneficios

- Crecimiento de la cuota de mercado

- $\quad$ Mejorar el posicionamiento del producto

- $\quad$ Penetración en un nuevo mercado

- $\quad$ Aumento de la satisfacción del cliente.

El ambiente de la mercadotecnia: Consiste en las fuerzas incontrolables que rodean a la compañía. Se debe partir del ambiente de la mercadotecnia, para luego buscar oportunidades y detectar amenazas. El entorno de la mercadotecnia está compuesto por todos los actores y las fuerzas que afectan la capacidad de la empresa para realizar transacciones efectivas con el mercado meta. El ambiente de mercadotecnia de la empresa se puede dividir en El Macro ambiente y El Microambiente.

- Macro ambiente: Son fuerzas que rodean a la empresa, sobre las cuales la misma no puede ejercer ningún control. Podemos citar el rápido cambio de tecnología, las tendencias demográficas, las políticas gubernamentales, la cultura de la población, la fuerza de la naturaleza, las tendencias sociales, etc.; fuerzas que, de una u otra forma pueden afectar significativamente y de las cuales la empresa puede aprovechar las oportunidades que ellas presentan y a la vez tratar de controlar las amenazas.

- Micro ambiente: Son todas las fuerzas que una empresa puede controlar y mediante las cuales se pretende lograr el cambio deseado. Entre ellas tenemos a los proveedores, la empresa en sí, según Philip Kotler también los competidores, intermediarios, clientes y públicos.

A partir del análisis del Microambiente nacen las fortalezas y las debilidades de la empresa. Son los elementos relacionados estrechamente con la empresa, marca todo lo que provienen desde el ofertante mismo".

\section{Pasos para realizar un plan de marketing}

Toda empresa, sin importar su tamaño o el sector en que se desenvuelve, precisa elaborar un plan de marketing para alcanzar el éxito. Lo más importante de un plan de marketing es que esté 
orientado a la acción de satisfacer una necesidad en el mercado y determine qué se quiere lograr, cuándo y cómo.

\section{Diagnóstico situacional}

El diagnostico situacional es el resultado de un proceso de investigación relacionado con la organización y el funcionamiento de las empresas, que permite determinar y evaluar las relaciones de causas-efectos de los problemas detectados y dar solución integral a los mismos.

\section{Análisis externo}

Un análisis externo consiste en detectar y evaluar acontecimientos y tendencias que suceden en el entorno de una empresa, que están más allá de su control y que podrían beneficiar o perjudicarla significativamente.

La razón de hacer un análisis externo es la de detectar oportunidades y amenazas, de manera que se puedan formular estrategias para aprovechar las oportunidades, y estrategias para eludir las amenazas o en todo caso, reducir sus consecuencias.

\section{Análisis interno}

Un análisis interno consiste en el estudio o análisis de los diferentes factores o elementos que puedan existir dentro de una empresa, con el fin de:

a) Evaluar los recursos con que cuenta una empresa para, de ese modo, conocer el estado o la capacidad con que cuenta.

b) Detectar fortalezas y debilidades, $y$, de ese modo, diseñar estrategias que permitan potenciar o aprovechar las fortalezas, y estrategias que permitan neutralizar o eliminar las debilidades

\section{Análisis FODA Matriz de las Fortalezas, Oportunidades, Debilidades Y Amenazas (FODA)}

La Matriz FODA es un marco conceptual para un análisis sistemático que facilita el aparecimiento entre las amenazas y oportunidades externas con las debilidades y fortalezas internas de la organización. 


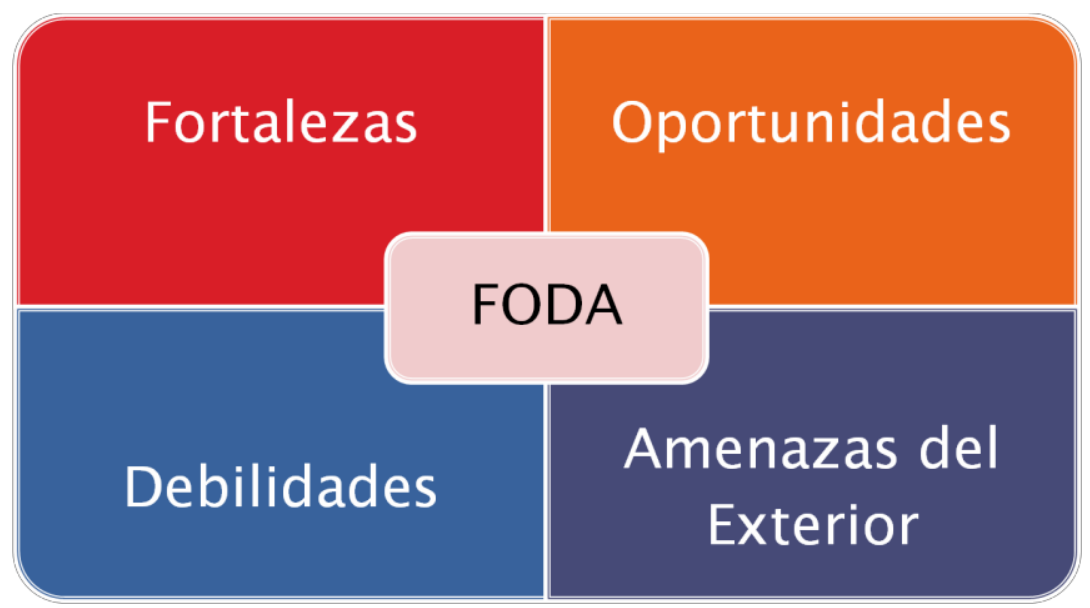

\section{Grafico 1. Matriz FODA}

La Matriz FODA, Fortalezas, Oportunidades, Debilidades Y Amenazas, es un método complementario de la evaluación interna EFI, de la evaluación externa EFE.

El análisis FODA nos ayuda a determinar si la organización está capacitada para desempeñarse en su medio. Mientras más competitiva en comparación con sus competidores este la empresa tendrá mayores probabilidades de éxito.

\section{Matriz de alto impacto}

Se puede definir como el enfrentamiento de factores internos y externos, con el propósito de generar estrategias alternativas La matriz F.O. D. A. es una importante herramienta de formulación de estrategias que conduce al desarrollo de cuatro tipos de estrategias.

a) Estrategias FO: Usan las fuerzas internas de la empresa para aprovechar la ventaja de las oportunidades externas.

Todos los gerentes querrán que sus organizaciones estuvieran en una posición donde pudieran usar las fuerzas internas para aprovechar las tendencias y hechos externos.

Cuando una organización enfrenta amenazas importantes, tratará de evitarlas para concentrarse en las oportunidades. 
b) Estrategias DO: Pretenden superar las debilidades internas aprovechando las oportunidades externas.

En ocasiones existen oportunidades externas clave, pero una empresa tiene debilidades internas que le impiden explotar dichas oportunidades.

c) Estrategias FA: Aprovechan las fuerzas de la empresa para evitar o disminuir el impacto de las amenazas externas; esto no quiere decir que una organización fuerte siempre deba enfrentar las amenazas del entorno externo.

d) Estrategias DA: Son tácticas defensivas que pretenden disminuir las debilidades internas y evitar las amenazas del entorno.

\section{Objetivos estratégicos}

Los objetivos establecen lo que se va a lograr y cuándo serán alcanzados los resultados. El objetivo es un propósito cuantificable que se desea alcanzar en un determinado tiempo.

La clave para el desarrollo de los objetivos estratégicos adecuados está en la identificación de los patrones de valor que las organizaciones proyectarán sobre sus segmentos de mercado objetivo.

Los objetivos estratégicos se deben formular para aprovechar las oportunidades, evitar las amenazas, impulsar las fortalezas y superar las debilidades.

Kotler \& Armstrong (2013) definen a la dirección de marketing como "el arte y la ciencia de elegir mercados meta con los cuales crear relaciones redituables. El objetivo de la dirección de marketing es encontrar, atraer, retener y aumentar los consumidores meta al crear, entregar y comunicar valor superior para el cliente". Continuando con los mismos autores, también afirman que:

"A un mayor conocimiento de las necesidades del consumidor en los nichos que atiende y a la reputación especial que adquiere se puede efectuar un marketing más eficaz (...) y promoverse de modo más eficiente al enfocar sus productos o servicios (...) hacia los consumidores a quienes puede servir mejor y de manera más redituable” (Kotler \& Armstrong, 2013: 57).

De la misma manera Kotler \& Lane Keller (2009) indican que "en la práctica, el marketing sigue un proceso lógico. El proceso de planeación de marketing consiste en identificar y analizar 
oportunidades de negocio, seleccionar los mercados meta, elaborar estrategias, definir programas y administrar el esfuerzo de marketing". Se coincide en que los mercados meta deben estar bien claros para dirigir estrategias enfocadas a lograr clientes satisfechos con productos modernos, de buena calidad, acorde a sus necesidades, con sinónimo de lujo y confort, la finalidad es conquistar nichos, y aprovechar las oportunidades que da el negocio.

Los autores antes mencionados acotan que "la dirección de marketing implica satisfacer los deseos y las necesidades de los consumidores. La función de cualquier empresa es ofrecer valor a sus clientes a cambio de utilidades".

\section{Conclusiones}

Para resumir, se puede afirmar que, el Plan de Marketing es una herramienta que, bien utilizada, permite a la empresa posicionarse en el mercado, de igual manera a su vez lograr un reposicionamiento exitoso según Sainz de Vicuña (2010) dice "el plan de marketing consiste en el proceso de definición (hoy) de lo que queremos ser en el futuro, apoyado en la correspondiente reflexión y pensamiento estratégico", la conceptualización permite a "Hogar 2000", asumir las estrategias para implementar un Plan de Marketing, junto al equipo de dirección de la empresa en los próximos meses con la finalidad de cumplir los objetivos, hacer una organización competitiva, con capacidad de crecimiento y rentabilidad en el mercado ecuatoriano.

Continuando con Sainz de Vicuña (2010) afirma que "es beneficioso que la empresa cuente con un plan siempre que se tenga la flexibilidad para ir adaptando la estrategia a los cambios que se vaya produciendo en el mercado". La recomendación es válida, sin embargo "Hogar 2000", ha dejado a un lado esta principal acción para poder competir, el estancamiento, y la poca adaptabilidad a los cambios del mercado de muebles y estantería es una de las causas por las que ha perdido posicionamiento y por ende su rentabilidad baje, además que la competencia se fortalezca al ofrecer mejores productos, y formas de comercialización.

En la misma dirección Ánzola (2012) expresa que "la empresa siempre necesita de la planeación" por lo tanto el Plan de Marketing requiere del compromiso y planificación para cumplir con los objetivos en pro de desarrollo y crecimiento de la empresa investigada. 
Además, la falta de visión global perjudica en ocasiones a muchas Pymes como afirma Sainz de Vicuña (2010) "se cierran puertas limitando su ámbito de actuación al mercado local. Este tipo de decisiones estratégicas pueden venir motivadas por una falta de autoconfianza o de visión empresarial" se debe tener en claro que las pymes deben asumir el reto que presenta el estar inmerso en el mundo globalizado, la expansión a mercados nacionales es el punto de partida para el enlace a mercados internacionales por ser el campo de crecimiento de la demanda en los mercados emergentes, "Hogar 2000" debe asumir el reto de crecer, en forma sostenida, a mercados como Centroamérica y El Caribe a fin explorar plazas que le otorguen oportunidades potenciales de crecimiento sin descuidar, cubrir y satisfacer las necesidades que tiene su mercado local.

\section{Referencias bibliográficas}

Alet José (2000) Como obtener Clientes Leales y Rentables. 1 Ed. España: Editorial Gestión.

Anzola, Servulo. (2012). Administración de pequeñas empresas (Vol. 2). México: McGraw-Hill.

Arellano C.R. (2000). Marketing, Enfoque América Latina. México: Mc Graw Hill.

Chiavenato. (2014). Introducción a la teoría de la Administración Interamericana. McGraw-Hill.

Chiavenato. H. (2010). Introducción de la Teoría General de la Administración (Vol. 7). México: McGraw-Hill.

Cohen, W. A. (2004). Plan de Mercadotecnia. México: Compañía Editorial Continental.

Conrad, Jay. (2009). Marketing de Guerrilla. México: Morgan James Publishing

Eguizábal, Raúl (1998). Historia de la Publicidad. España: Celeste Ediciones

Fernández V. R. (2001). Manual para Elaborar un Plan de Mercadotecnia: Un Enfoque Latinoamericano. México: Thomson.

Ferrel O.C \& Hartline Michael D. (2012). Estrategia de Marketing. 5ta Ed. México Distrito Federal: Cengage Learning.

Ferrell, H. A. (2009). Introducción a los Negocios en un Mundo Cambiante (Vol. 9). México: McGraw-Hill. 
Fischer L. \& Espejo J. (2004). Mercadotecnia. México: Mc Graw Hill.

Fischer, Laura \& Espejo, Jorge (2011). Mercadotecnia. Cuarta edición. México: McGraw-Hill.

Kootnz H. \& Weihrich. (1998). Administración, una perspectiva global. México: Mc Graw Hill.

Kotler \& Armstrong (2010). Fundamentos del Marketing. España: Pearson Educación.

Kotler, P. \& Armstrong, G. (2001). Segmentación de mercados, mercados meta y

Kotler, P., Bowen, J. \& Makens, J. (1997). Mercadotecnia para Hotelería y Turismo. México:

Prentice Hall Hispanoamérica.

Kotler, Philip \& Armstrong Gary (2012). Marketing. Décimocuarta edición. México: Pearson Educación.

Lane Keller, Kevin (2008). Administración Estratégica de Marca, Branding. 3era. Edición. México: Pearson Educación.

McCarthy, E. J. (1984). Basic Marketing: a managerial approach. México: Cengage Learning Editores.

McIntosh, R. W., Goeldner, C. R. \& Brent, R.J.R. (2001). Turismo: Planeación, Administración y Perspectivas. México: Limusa.

Posicionamiento para obtener una ventaja competitiva. México: Prentice Hall Hispanoamérica.

Prawda, W. J. (2000). Métodos y Modelos de Investigaciones de operaciones. México: Limusa.

Riano Claudio (1990). El Marketing Mix, Conceptos Estrategias y Aplicaciones. $1^{\text {o }}$ Ed. España: Mapcal Editorial.

Ries, A. \& Trout, J. (2003). Posicionamiento: La batalla por sus mentes. México: McGraw Hill. Robbins \& Coulter. (2005). Administración. España- Pearson Educación. 108

Rodríguez, J. (2000). Administración de pequeñas y medianas empresas (Vol. 5). México: Thomson.

Rojas Risco, Demóstenes (2013). La Biblia del Marketing. España: Lexus Editores. 
Kelly J. Estrada Realpe, Rugina Elidea Quiñonez, Luz M. Cifuentes Quiñonez, Jorgeli Ayoví Caicedo

Sainz de Vicuña (2012) El plan de marketing estratégico. España: ESIC Editorial

Sapag Nassir, Sapag Reinaldo (2010) Preparación y Evaluación de Proyectos. 4ta. Edición. Chile: Mc. Graw Hill.

Stanton et al (2009). Fundamentos del Marketing. México: Mc. Graw Hill.

Trout \& Rivkin. (2014). "El nuevo posicionamiento". México: Ed. Limusa.

Zikmund, William G. (1998). Investigación de mercados. México: Prentice Hall Interamericana. 